$\Rightarrow$ CELL DEATH

\title{
Programmed necrosis: putting the pieces together
}

\section{ce}

the

recruitment

and activation

of DRP1 by

PGAM5S is

crucial for the

execution of

necroptosis
Necroptosis (also known as programmed necrosis) occurs following the activation of receptor-interacting protein 1 (RIP1; also known as RIPK1) in response to the ligation of tumour necrosis factor receptor (TNFR) and the formation of a RIP1-RIP3 complex (necrosome). However, the events that mediate necroptosis downstream of necrosome formation remain largely elusive. Two recent studies by Wang and colleagues delineate the effectors of the necroptotic cell death pathway downstream of RIP3 activation.

While screening 200,000 chemical compounds, Sun et al. identified necrosulfonamide (NSA) as a potent inhibitor of necroptosis that acts downstream of necrosome formation and the phosphorylation of RIP3 and RIP1. Experiments with this newly identified inhibitor revealed that it directly targets mixed lineage kinase domain-like protein (MLKL). MLKL, which is a kinase-dead protein, was found to be recruited to the necrosome following RIP3 activation.

So what is the role of MLKL in necroptosis? Interestingly, although MLKL knockdown did not prevent

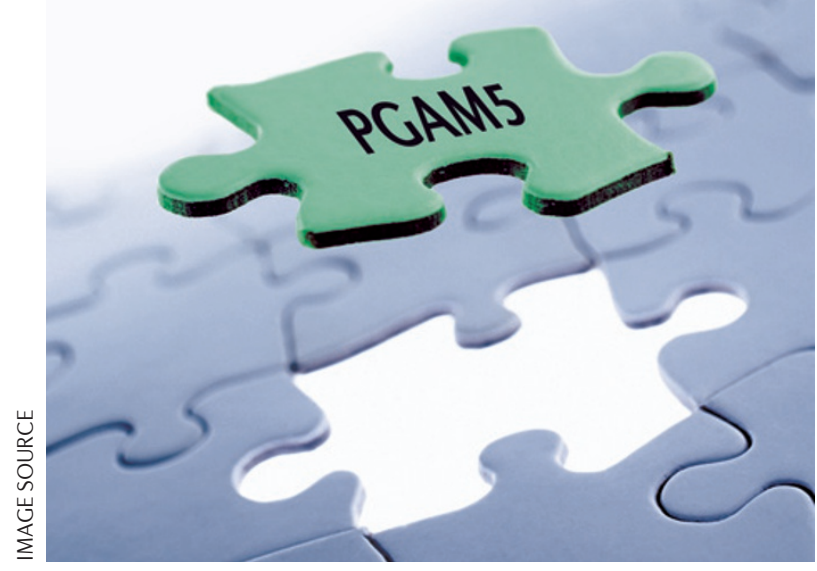

RIP1 and RIP3 phosphorylation in response to necroptosis-inducing factors, it increased cell survival. The kinase-like domain of MLKL and the kinase domain of RIP3 were found to be essential for MLKL recruitment to the necrosome, which in turn facilitated RIP3 autophosphorylation. Autophosphorylated RIP3 was shown to phosphorylate MLKL on Thr357 and Ser358 of the kinase-like domain, and MLKL phosphorylation by RIP3 was indispensable for programmed necrosis. As MLKL lacks any kinase activity, Sun et al. suggest that its phosphorylation may facilitate its interaction with downstream effectors.

Wang et al. also used NSA, in this case to identify the downstream effectors of the RIP1-RIP3-MLKL complex in necroptotic cells. They observed that the mitochondrial phosphatase PGAM5 (which is normally localized on the outer mitochondrial membrane) co-precipitated with RIP3 under necroptosis-inducing conditions, which also led to PGAM5 phosphorylation by the RIP3 complex and consequent increased PGAM5 phosphatase activity. This indicates that PGAM5 is a necrosome substrate.

There are two splice variants of PGAM5, with the shorter isoform (PGAM5S) being more hydrophobic than the longer isoform (PGAM5L). Small interfering RNA studies revealed that both isoforms are essential for necroptosis, with PGAM5L being initially recruited to the RIP1-RIP3-MLKL complex, followed by PGAM5S. The recruitment of both PGAM5 isoforms to the necrosome resulted in their phosphorylation. However, only PGAM5S recruitment and phosphorylation depended on the presence of MLKL and was sensitive to inhibition by NSA. Based on these findings, Wang et al. suggest that PGAM5S is the central downstream effector of the MLKL-containing necrosome.

Confocal microscopy revealed that cells undergoing necroptosis exhibit mitochondrial fission, which was also sensitive to NSA treatment. So, Wang et al. hypothesized that the presence of the hydrophobic PGAM5S in the necrosome might enable translocation of the necrosome to a more hydrophobic environment, such as the one found at constriction sites where mitochondrial fission is initiated. Indeed, recruitment of PGAM5S to the necrosome and its subsequent phosphorylation coincided with a shift of phosphorylated RIP1, RIP3, MLKL and PGAM5L to a more hydrophobic environment, which also contained dynamin-related protein 1 (DRP1), the GTPase that controls mitochondrial fission. Strikingly, DRP1 was found to be recruited to the necrosome by PGAM5S and to be subsequently activated through the PGAM5S phosphatase activity. Moreover, knockdown of PGAM5S or DRP1 prevented programmed necrosis, indicating that the recruitment and activation of DRP1 by PGAM5S is crucial for the execution of this pathway.

Together, the two studies identify crucial components of the programmed necrosis pathway. Interestingly, Wang et al. observed that PGAM5 is required not only for TNFR-triggered necroptosis but also for intrinsic necrosis induced by reactive oxygen species. Thus, PGAM5 might be located at the convergence point of the necrotic pathways. Maria Papatriantafyllou

ORIGINAL RESEARCH PAPERS Sun, L. et al. Mixed lineage kinase domain-like protein mediates necrosis signalling downstream of RIP3 kinase. Cell 148, 213-227 (2012) | Wang, Z. et al. The mitochondrial phosphatase PGAM5 functions at the convergence point of multiple necrotic death pathways. Cell 148, 228-243 (2012) 


\section{Correction}

The last two sentences of this highlight have been corrected to read "Interestingly, Wang et al. observed that PGAM5 is required not only for TNFR-triggered necroptosis but also for intrinsic necrosis induced by reactive oxygen species. Thus, PGAM5 might be located at the convergence point of the necrotic pathways." 